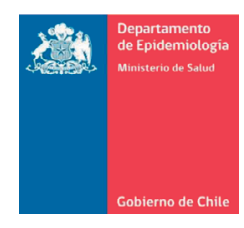

\title{
Informe Anual 2016 Situación epidemiológica de gonorrea (CIE 10: A54)
}

\author{
Informe elaborado por: Karen Cáceres ${ }^{1}$ \\ Con colaboración de: \\ Programa de Prevención y Control del VIH y las ITS, \\ Departamento de Salud y Pueblos Indígenas e Interculturalidad \\ Unidad de migración y salud
}

Edición a cargo de: Patricia Salvadó

\section{Anual Report Epidemiology of gonorrohea (CIE 10: A54)}

Gonorrhea is produced by the bacterium Neisseria gonorrhoeae, which is capable of infecting different types of mucous membranes depending on sexual practices. It is located preferably in the man's urethra and in the woman's cervix, also in the rectum and pharynx. Its main form of transmission is through unprotected sex and, occasionally, through the birth canal in newborns. This report presents the epidemiological situation of gonorrhea until 2016 in Chile. A descriptive analysis of the cases that entered the surveillance was carried out. Since 2000, there has been a progressive trend towards decreasing gonorrhea rates; However, in 2015 and 2016 there was an increase in the rate in relation to 2014. In 2016, 2,039 cases were presented, representing an increase of $38 \%$ with respect to the median of the previous five-year period (1,473 cases). Regarding the age of the cases, the greatest risk was found in the group of 15 to 24 years, highlighting that, as of 2013, the group rate of 15 to 19 years exceeded the group of 25 to 29 years. According to geographical distribution, the highest reporting rates were in the regions of the extreme north and south of the country, with the highest risk regions being Tarapacá, Antofagasta, Los Lagos and Aysén.

Key words: sexually transmitted infections; gonorrhea; Neisseria gonorrhoeae; epidemiology.

Palabras clave: infecciones de transmisión sexual; gonorrea; Neisseria gonorrhoeae; epidemiología.

\section{Antecedentes}

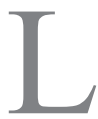
as infecciones de transmisión sexual (ITS) son una de las principales causas de enfermedad aguda, infertilidad, discapacidad a largo plazo y muerte en el mundo ${ }^{1}$.

La gonorrea, también conocida como blenorragia, es producida por la bacteria Neisseria gonorrhoeae. Ésta es capaz de infectar diferentes tipos de mucosas, de preferencia la uretra en el hombre y el cuello uterino en la mujer, pudiendo además encontrarse en el recto, conjuntiva, faringe, vulva y vagina de la mujer, dependiendo de las prácticas sexuales ${ }^{2}$. Su principal forma de transmisión es por relaciones sexuales no protegidas y ocasionalmente a través del conducto del parto en los recién nacidos $(\mathrm{RN})$.

Entre las complicaciones de esta infección, en el hombre es posible encontrar abscesos prostáticos y epididimitis que pueden llevar a la infertilidad. En mujeres puede causar enfermedad inflamatoria pélvica con consecuencias como infertilidad, embarazo ectópico y dolor pélvico crónico. Estudios epidemiológicos y biológicos han proporcionado evidencia de que las infecciones gonocóccicas además facilitan la transmisión del virus de inmunodeficiencia humana (VIH) $)^{2,3,4}$.

En general, esta infección produce mayor sintomatología en hombres, lo que permite la consulta y tratamiento oportunos; en cambio, en las mujeres las manifestaciones son mayoritariamente asintomáticas, hasta que se producen las complicaciones.

La gonorrea es de vigilancia universal según el Decreto Supremo $N^{\circ} 158 / 2004$; por lo tanto, el médico tratante debe notificar según se establece en la definición de caso. Además, $N$. gonorrhoeae es un agente de vigilancia de laboratorio para monitorizar su resistencia a antimicrobianos ${ }^{5}$.

El propósito de la vigilancia epidemiológica es entregar información que permita orientar la toma de decisiones y la planificación de estrategias de prevención y control; en este sentido, el objetivo de este informe es describir la tendencia y el comportamiento de los casos de gonorrea en Chile hasta el año 2016.
'Departamento de Epidemiología División de Planificación Sanitaria. Ministerio de Salud de Chile.

Recibido: 7 de abril de 2018

Correspondencia a: Karen Cáceres Burton karen.caceres@minsal.cl 


\section{Método}

El presente informe es un análisis de tipo descriptivo de los casos confirmados de gonorrea en Chile (código CIE10: A54) hasta el año 2016. Para su elaboración se utilizó la información proveniente de bases de datos secundarias:

- Base de datos de Enfermedades de Notificación Obligatoria (ENO) dependiente del Departamento de Epidemiología del Ministerio de Salud de Chile (MINSAL) y del Departamento de Estadísticas en Salud (DEIS). Esta información se encuentra validada hasta el año 2015, mientras que la del año 2016 corresponde a información provisoria.

- Base de datos de defunciones obtenidas desde el Departamento de Estadísticas e Información en Salud (DEIS) del MINSAL, disponibles hasta el año 2014.

- Base de datos de egresos hospitalarios obtenidas desde el DEIS del MINSAL, disponibles hasta el año 2014.

Se presentan indicadores descriptivos para el análisis por tiempo, lugar, persona (sexo, edad, clasificación de la infección por CIE-10) y determinantes sociales (nacionalidad y pertenencia a pueblos originarios). Se calcularon porcentajes, medianas y tasas. Estas últimas se amplificaron por cien mil habitantes (habs.) utilizando las proyecciones de población del Instituto Nacional de Estadística (INE) del año 2012. Se usaron gráficos y tablas para presentar los resultados utilizando el software Microsoft Excel. Para el análisis de la información territorial, se elaboraron cartografías utilizando el software ArcGIS 10.0.

Se calculó el Índice epidémico acumulado para el año 2016, que es la razón entre los casos acumulados a un tiempo determinado y los casos acumulados esperados (mediana del quinquenio) para el mismo período. Si el cociente se encuentra entre $0,76-1,24$, la incidencia es normal; si es menor o igual a 0,75 es baja y si es mayor o igual a 1,25, la incidencia es alta.

Se definió como caso de gonorrea a la presencia de examen de laboratorio microbiológico, inmuno-enzimático o de biología molecular que señale infección por $N$. gonorrhoeae, con y sin sintomatología o antecedente de contacto con un caso confirmado ${ }^{5}$.

Con relación a la Ley $\mathrm{N}^{\circ} 20.584$ que "Regula los derechos y deberes que tienen las personas en relación con acciones vinculadas a su atención en salud", este informe no vulnera la mencionada ley, debido a que utiliza datos del Ministerio de Salud, los que se recogen dando cumplimiento a su rol según las siguientes leyes previas: DFL N ${ }^{\circ} 1 / 2005$ del MINSAL y Ley $N^{\circ} 19.628$ sobre datos sensibles.

\section{Resultados}

\section{Situación epidemiológica}

Desde el año 2000, se observa una tendencia progresiva a la disminución de las tasas, estabilizándose entre los años 2008 y 2009. En el año 2015 comenzó un ascenso (10,0 por cien mil habs.) (Figura 1 y Tabla 1). En el año 2016 se presentaron 2.039 casos, lo que representó 38\% más con respecto a la mediana del quinquenio anterior ( 1.473 casos) y cuyo índice epidémico acumulado es de 1,4 , lo que indica que en ese año la cantidad de casos se encuentra por sobre lo esperado para este período.

Desde el año 2007, se observa una tendencia sostenida al alza de las tasas en el grupo etario entre 15 y 39 años. En el año 2016 este grupo de edad representó 87\% del total de los casos.

En el quinquenio 2012-2016, las tasas más elevadas de gonorrea se presentaron en el grupo de 15 a 24 años,
Figura 1. Tasa de notificación de gonorrea. Chile 2000-2016*. *2016 datos provisorios Fuente: Depto. Epidemiología, DEIS. DIPLASMinisterio de Salud de Chile.

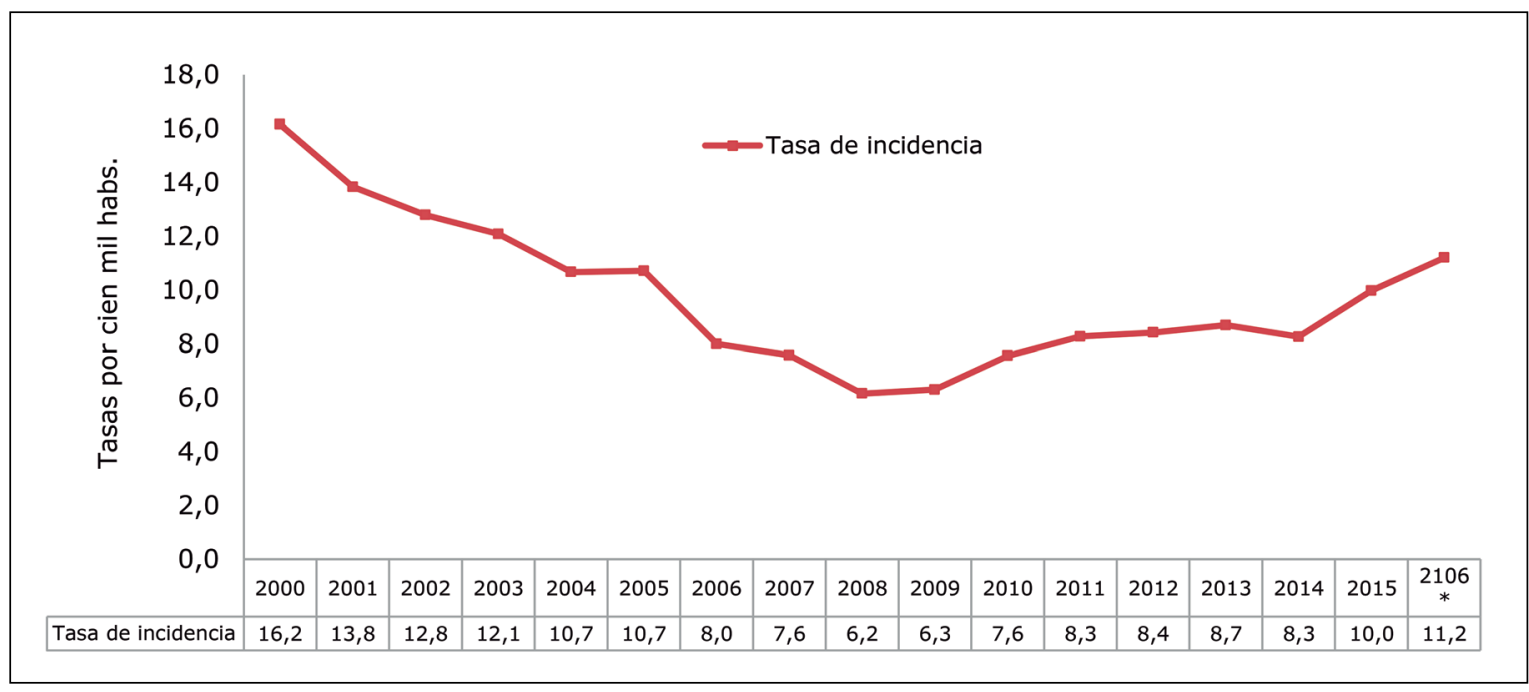




\begin{tabular}{ccc}
\hline Tabla 1. Casos y tasas de gonorrea. Chile $\mathbf{2 0 0 0 - 2 0 1 6 ^ { * }}$ \\
\hline Años & Casos & Tasas/100.000 hbs. \\
\hline 2000 & 2.488 & 16,2 \\
\hline 2001 & 2.385 & 13,8 \\
\hline 2002 & 2.014 & 12,8 \\
\hline 2003 & 1.918 & 12,1 \\
\hline 2004 & 1.718 & 10,7 \\
\hline 2005 & 1.720 & 10,7 \\
\hline 2006 & 1.293 & 8,0 \\
\hline 2007 & 1.251 & 7,6 \\
\hline 2008 & 1.028 & 6,2 \\
\hline 2009 & 1.065 & 6,3 \\
\hline 2010 & 1.290 & 7,6 \\
\hline 2011 & 1.428 & 8,3 \\
\hline 2012 & 1.470 & 8,4 \\
\hline 2013 & 1.533 & 8,7 \\
\hline 2014 & 1.473 & 8,3 \\
\hline 2015 & 1.797 & 10,0 \\
\hline $2016^{*}$ & 2.039 & 11,2 \\
\hline
\end{tabular}

*2016, datos provisorios. Fuente: Base de datos ENO. DEIS. Dpto. Epidemiología, MINSAL, Chile. observándose el mayor aumento en el año 2016. Específicamente en el grupo quinquenal de 20 a 24 años, aumentó su tasa desde el año 2012 de 28,1 por cien mil habitantes (habs.) a 41,5 por cien mil habs. Se destaca que a partir del año 2013 se observó un desplazamiento de la infección a edades menores, y es así como la tasa del grupo de 15 a 19 años superó a la del grupo de 25 a 29 años. El grupo de adolescentes mostró tasas que oscilaron entre 18,4 (2012) y 36,5 (2016) por cien mil habs (Figura 2, Tabla 2). Debido a este cambio, se produjo una leve baja en la mediana de edad de incidencia de la infección, desde 25 años en el 2012 a 24 años en el 2016.

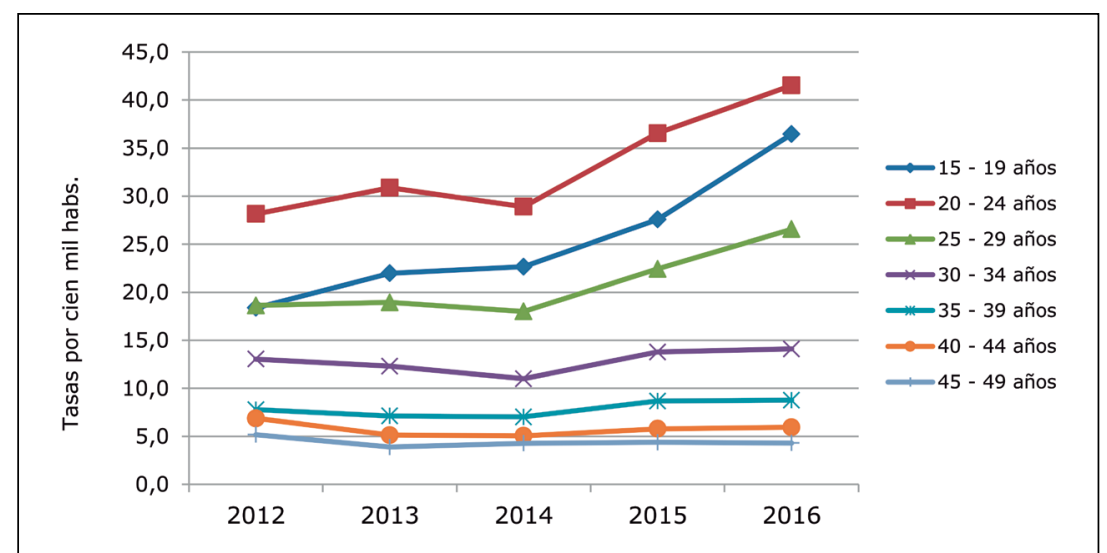

Figura 2. Tasas de gonorrea según grupos de edad. Chile 2011-2016*. *2016 datos provisorios. Fuente: Depto. Epidemiología, DEIS. DIPLAS-Ministerio de Salud de Chile.

\begin{tabular}{|c|c|c|c|c|c|c|c|c|c|c|}
\hline \multirow{2}{*}{$\begin{array}{l}\text { Grupo de } \\
\text { edad }\end{array}$} & \multicolumn{2}{|c|}{2012} & \multicolumn{2}{|c|}{2013} & \multicolumn{2}{|c|}{2014} & \multicolumn{2}{|c|}{2015} & \multicolumn{2}{|c|}{$2016^{*}$} \\
\hline & Casos & Tasas & Casos & Tasas & Casos & Tasas & Casos & Tasas & Casos & Tasas \\
\hline 00 - 04 años & 7 & 0,6 & 7 & 0,6 & 9 & 0,7 & 7 & 0,6 & 14 & 1,1 \\
\hline 05 - 09 años & 8 & 0,7 & 6 & 0,5 & 3 & 0,2 & 10 & 0,8 & 6 & 0,5 \\
\hline $10-14$ años & 6 & 0,5 & 14 & 1,1 & 9 & 0,7 & 9 & 0,7 & 16 & 1,3 \\
\hline $15-19$ años & 256 & 18,4 & 301 & 22,0 & 305 & 22,7 & 365 & 27,6 & 474 & 36,5 \\
\hline 20 - 24 años & 421 & 28,1 & 461 & 30,9 & 428 & 28,9 & 534 & 36,6 & 597 & 41,5 \\
\hline 25 - 29 años & 262 & 18,6 & 273 & 18,9 & 265 & 18,0 & 336 & 22,4 & 403 & 26,6 \\
\hline 30 - 34 años & 164 & 13,0 & 158 & 12,3 & 145 & 11,0 & 187 & 13,8 & 197 & 14,1 \\
\hline 35 - 39 años & 96 & 7,8 & 88 & 7,1 & 87 & 7,0 & 108 & 8,7 & 110 & 8,8 \\
\hline 40 - 44 años & 86 & 6,9 & 64 & 5,1 & 63 & 5,1 & 72 & 5,8 & 74 & 5,9 \\
\hline 45 - 49 años & 65 & 5,2 & 49 & 3,9 & 54 & 4,3 & 55 & 4,4 & 54 & 4,3 \\
\hline 50 - 54 años & 33 & 2,9 & 41 & 3,5 & 52 & 4,3 & 46 & 3,8 & 39 & 3,2 \\
\hline 55 - 59 años & 30 & 3,2 & 30 & 3,1 & 27 & 2,7 & 32 & 3,0 & 29 & 2,7 \\
\hline 60 - 64 años & 12 & 1,6 & 23 & 3,0 & 14 & 1,8 & 18 & 2,2 & 10 & 1,2 \\
\hline 65 - 69 años & 12 & 2,1 & 10 & 1,7 & 3 & 0,5 & 5 & 0,8 & 10 & 1,5 \\
\hline 70 - 74 años & 5 & 1,2 & 3 & 0,7 & 5 & 1,1 & 7 & 1,4 & 2 & 0,4 \\
\hline 75 - 79 años & 5 & 1,6 & 3 & 0,9 & 1 & 0,3 & 3 & 0,9 & 1 & 0,3 \\
\hline 80 y más años & 2 & 0,6 & 2 & 0,6 & 3 & 0,8 & 2 & 0,5 & 3 & 0,7 \\
\hline
\end{tabular}

*2016 datos provisorios. Tasas por cien mil habs. Fuente: Depto. Epidemiología, DEIS. DIPLAS - Ministerio de Salud de Chile. 


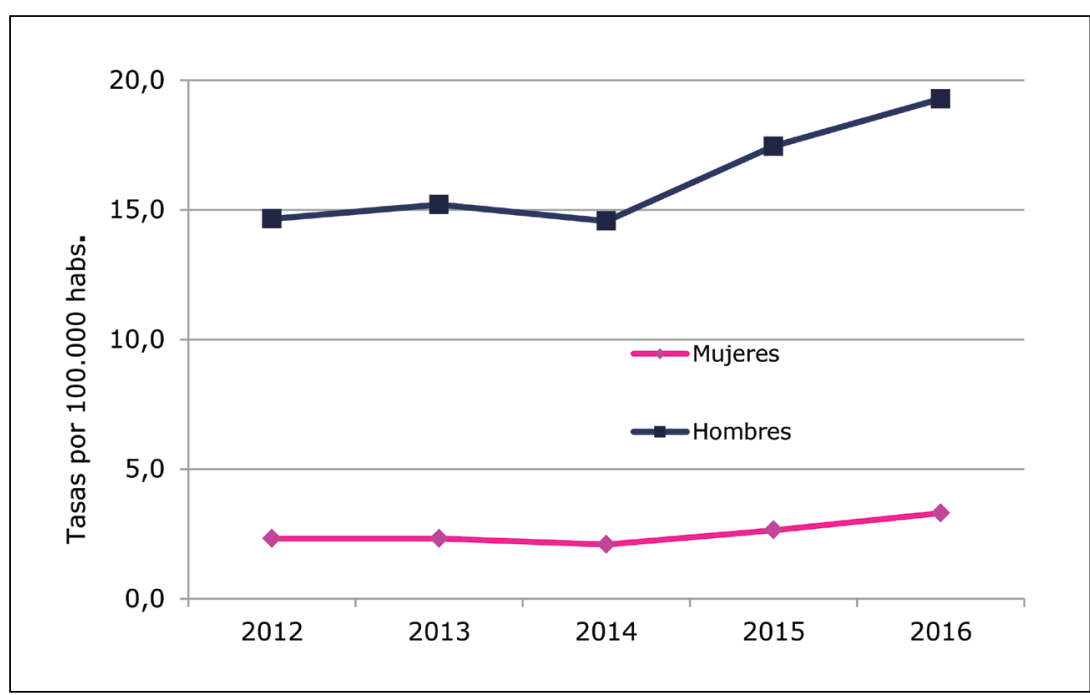

Figura 3. Tasas de gonorrea según sexo. Chile 2012-2016* * 2016 datos provisorios. Fuente: Depto. Epidemiología, DEIS. DIPLAS-Ministerio de Salud de Chile.

\begin{tabular}{ccccc} 
Tabla 3. Casos y tasas de gonorrea según sexo. Chile 2012-2016* \\
\hline Años & Casos & Hombres & Mujeres \\
& 1.266 & 14,7 & Casos & Tasas \\
2012 & 1.327 & 15,2 & 204 & 2,3 \\
2013 & 1.285 & 14,6 & 206 & 2,3 \\
2014 & 1.556 & 17,5 & 188 & 2,1 \\
2015 & 1.736 & 19,3 & 240 & 2,6 \\
$2016^{*}$ & & & 303 & 3,3
\end{tabular}

*2016 datos provisorios. Tasas por cien mil habs. Fuente: Depto. Epidemiología, DEIS. DIPLASMinisterio de Salud de Chile. riesgo entre los 15 y 29 años. Sin embargo, las mayores tasas según grupo quinquenal, se registraron entre los 15 y 19 años. Destaca que a partir de los 55 años en adelante se presentaron muy pocos casos de gonorrea en mujeres (mediana de 7 casos anuales).

Las mujeres gestantes representaron 3\% del total de casos de gonorrea notificados en mujeres en el período de estudio, con una mediana de ocho gestantes anuales.

Con relación a la clasificación del tipo de gonorrea, en el quinquenio 2012-2016, 53\% fue notificado como infección gonocóccica, no especificada (A54.9), 45\% se notificó como infección gonocóccica del tracto urinario (A54.0-A54.2). La infección gonocóccica del ojo y la del recto y ano representaron cada una $0,2 \%$ y $1 \%$ correspondió a faringitis gonocóccica. El $67 \%$ de los casos de gonorrea oftálmica se notificó en recién nacidos.

\section{Dinámica espacial}

Según distribución geográfica, en el último quinquenio las mayores tasas de notificación se presentaron en las regiones del extremo norte y sur del país.

Las regiones de Tarapacá, Antofagasta, Los Lagos, Aysén y Arica y Parinacota mostraron los mayores riesgos. El aumento de casos que se presentó en estas regiones se concentró en el grupo de 15 a 24 años.

Las regiones del centro sur del país (de la Región Metropolitana a Los Ríos) presentaron tasas inferiores al valor nacional en este período (Figura 4 y Tabla 4).

Destaca que, a partir del año 2011, la Región de Aysén comenzó a mostrar un gran número de casos, presentando tasas de entre 38,3 y 48,0 por cien mil habs. Ante esta situación, la región desarrolló acciones integradas para la prevención y control de las ITS, mostrando en el año 2015 una disminución de las tasas; no obstante, en el 2016 volvió a presentar un alza de 38,4 por cien mil habs.

En el año 2016, la Región de Tarapacá presentó la mayor tasa nacional superando en cinco veces la tasa del país. Le siguieron las regiones de Los Lagos, Aysén, Antofagasta y Arica y Parinacota, todas sobrepasando la tasa de Chile de 11,2 por cien mil habs (Tabla 4). Las regiones de Tarapacá, Antofagasta y Araucanía ese año doblaron el número de casos esperados, según la mediana del quinquenio anterior.

\section{Determinantes sociales}

\section{Nacionalidad}

En el quinquenio 2012-2016, 3,6\% del total de casos de gonorrea se notificaron en personas extranjeras, siendo $83 \%$ de sexo masculino. El año 2016 se presentó un aumento en el porcentaje de extranjeros notificados, representando $6,7 \%$ del total de casos.

El mayor porcentaje de casos notificados en migrantes se encuentra en las regiones de Tarapacá (38\%), Antofagasta (24\%) y la R. Metropolitana (24\%). 


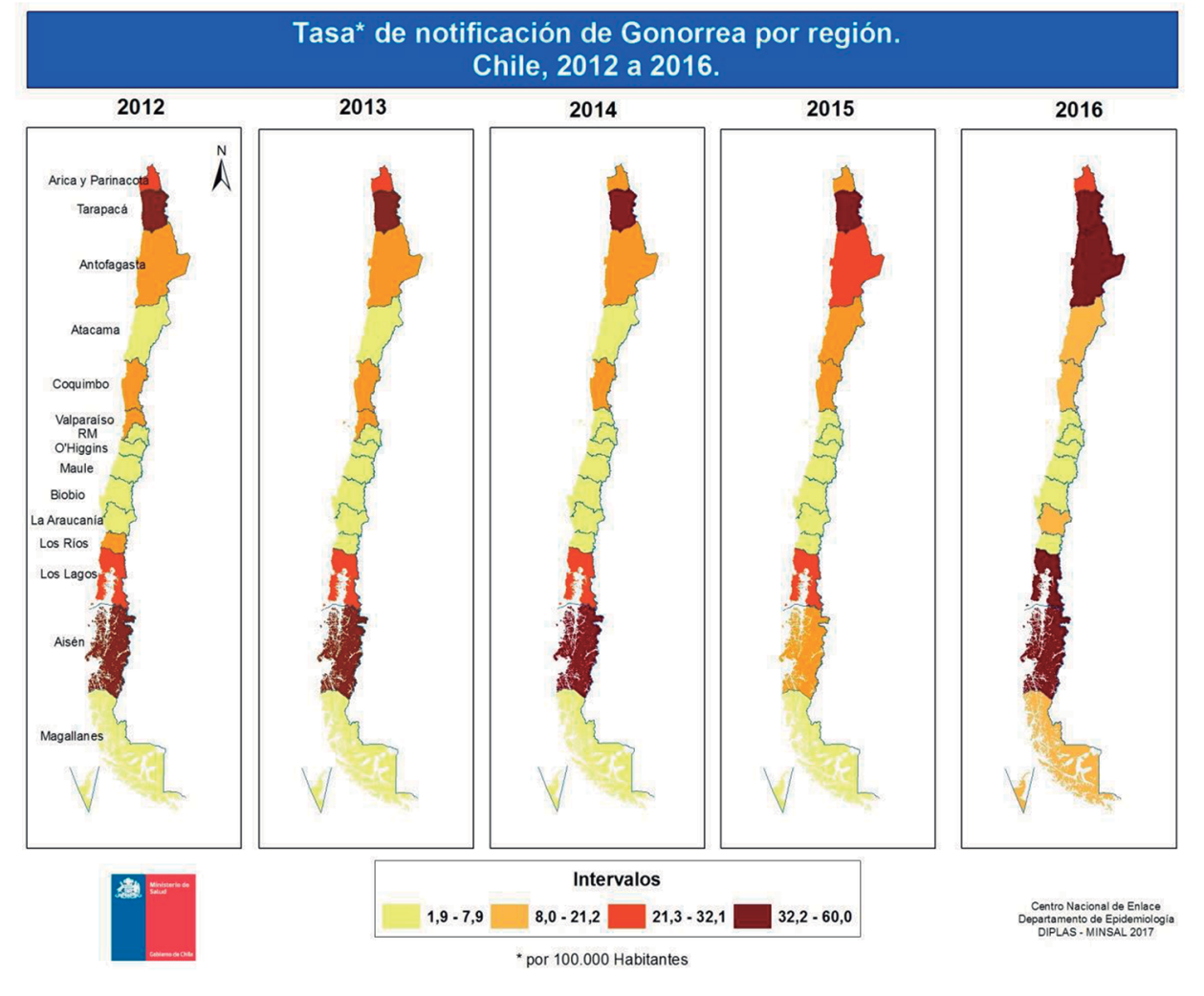

Figura 4. *2016 datos provisorios. Tasas por cien mil habs. Fuente: Depto. Epidemiología, DEIS. DIPLAS Ministerio de Salud de Chile.

\begin{tabular}{|c|c|c|c|c|c|c|c|c|c|c|}
\hline \multirow[t]{2}{*}{ Regiones } & \multicolumn{2}{|c|}{2012} & \multicolumn{2}{|c|}{2013} & \multicolumn{2}{|c|}{2014} & \multicolumn{2}{|c|}{2015} & \multicolumn{2}{|c|}{$2016^{*}$} \\
\hline & Casos & Tasas & Casos & Tasas & Casos & Tasas & Casos & Tasas & Casos & Tasas \\
\hline Arica y Parinacota & 56 & 24,7 & 50 & 21,6 & 35 & 14,9 & 41 & 17,1 & 66 & 27,1 \\
\hline Tarapacá & 104 & 33,2 & 116 & 36,2 & 177 & 53,8 & 181 & 53,7 & 207 & 60,0 \\
\hline Antofagasta & 68 & 11,4 & 102 & 16,9 & 117 & 19,1 & 198 & 31,8 & 218 & 34,5 \\
\hline Atacama & 16 & 5,3 & 23 & 7,6 & 15 & 4,9 & 37 & 11,8 & 27 & 8,5 \\
\hline Coquimbo & 101 & 13,7 & 93 & 12,4 & 93 & 12,2 & 139 & 18,0 & 86 & 11,0 \\
\hline Valparaíso & 151 & 8,5 & 176 & 9,8 & 124 & 6,9 & 128 & 7,0 & 126 & 6,8 \\
\hline Metropolitana & 416 & 5,9 & 453 & 6,3 & 396 & 5,5 & 440 & 6,0 & 513 & 6,9 \\
\hline O'Higgins & 32 & 3,6 & 42 & 4,7 & 36 & 4,0 & 38 & 4,1 & 46 & 5,0 \\
\hline Maule & 20 & 2,0 & 29 & 2,8 & 41 & 4,0 & 63 & 6,0 & 58 & 5,5 \\
\hline Biobío & 145 & 7,0 & 109 & 5,2 & 125 & 6,0 & 164 & 7,8 & 164 & 7,7 \\
\hline Araucanía & 47 & 4,8 & 57 & 5,8 & 35 & 3,6 & 63 & 6,4 & 102 & 10,2 \\
\hline Los Ríos & 39 & 9,9 & 25 & 6,3 & 27 & 6,7 & 25 & 6,2 & 26 & 6,4 \\
\hline Los Lagos & 217 & 26,4 & 197 & 23,8 & 197 & 23,6 & 252 & 30,0 & 344 & 40,6 \\
\hline Aysén & 46 & 43,6 & 51 & 48,0 & 51 & 47,5 & 23 & 21,2 & 42 & 38,4 \\
\hline Magallanes & 12 & 7,4 & 10 & 6,1 & 4 & 2,4 & 5 & 3,0 & 14 & 8,5 \\
\hline Total & 1.470 & 8,4 & 1.533 & 8,7 & 1.473 & 8,3 & 1.797 & 10,0 & 2.039 & 11,2 \\
\hline
\end{tabular}


Según procedencia, $89 \%$ proviene de América del Sur y $5 \%$ de América Central.

\section{Pertenencia a pueblos originarios}

Se observa una relativa estabilidad de casos que declaran pertenencia a pueblos originarios entre los años 2012 y 2016 con una mediana de cinco casos anuales. El $81 \%$ de los notificados fueron hombres. Del total de notificaciones en el quinquenio, $0,3 \%(\mathrm{n}=27)$ de los casos declaró pertenecer a un pueblo originario.

\section{Mortalidad y egresos hospitalarios}

Según los registros de defunción, se informa sólo un fallecimiento por gonorrea en el año 2005, que corresponde a un hombre de 83 años. Desde ese año en adelante no se han registrado otros fallecidos por esta causa.

En el período 2010 a 2014, la infección gonocóccica representó $0,04 \%$ del total de egresos hospitalarios, siendo $58 \%$ de los casos hospitalizados por gonorrea, mujeres. El $48 \%$ del total de casos tuvo como diagnóstico de egreso una infección gonocóccica del tracto genitourinario inferior y $16 \%$ pelvi-peritonitis y otras infecciones genitourinarias. Con relación a la edad, $44 \%$ tenía entre 30 y 49 años y $15 \%$ tenía menos de 5 años de edad.

\section{Discusión}

Gonorrea es una ITS curable; no obstante, según estima la Organización Mundial de la Salud (OMS), su prevalencia global e incidencia siguen siendo altas, presentando 78 millones de nuevos casos anuales ${ }^{6}$. Las ITS suelen afectar principalmente a población entre 15 a 24 años que constituyen sólo $25 \%$ de la población sexualmente activa; sin embargo, concentran $50 \%$ de los nuevos casos de ITS reportadas en el mundo ${ }^{7}$. En Chile, según los resultados de vigilancia se presenta un aumento de la tasa de incidencia durante los últimos dos años, que se focaliza principalmente en el grupo de 15 a 24 años.

En este contexto, es relevante considerar que la infección gonocóccica frecuentemente es transmitida por personas que cursan asintomáticas o que presentan síntomas leves de la infección. Estos individuos son importantes, porque frecuentemente no acuden a la atención de salud, no reciben tratamiento y continúan sexualmente activos, facilitando la transmisión de esta infección.

Entre los hombres puede producir síntomas que le inducen a buscar tratamiento curativo lo suficientemente pronto para evitar las secuelas, pero a menudo no es lo tan pronto como para prevenir la transmisión a otros, no es lo suficientemente oportuno. Entre las mujeres, las infecciones gonocóccicas normalmente son asintomáticas o pueden no producir síntomas reconocibles hasta que se han producido complicaciones ${ }^{8}$, lo que dificulta el diagnóstico oportuno.
Se ha determinado que la existencia de co-infecciones de transmisión sexual aumentan la capacidad infectante de VIH, favorecido por los procesos inflamatorios locales que se producen en la gonorrea. Según Kalichman, la prevalencia de ITS fue mayor en el momento del diagnóstico de VIH, lo que refleja el papel de las ITS en la transmisión del VIH ${ }^{9}$

Según un análisis multivariado realizado en relación a factores de riesgo e ITS, sólo ser más joven estaba fuertemente asociado a tener múltiples ITS. Para el caso de la gonorrea, por cada año que aumentaba la edad de tamizaje, el riesgo de infección disminuía en 6\% (CI 95\%: 4-9) ${ }^{10}$.

Como se mencionó, en el país muestra una tasa ascendente de esta ITS en la población de 15 a 24 años, lo que es un potente indicador de que existe un aumento de otras ITS en ellos. (VIH, sífilis, hepatitis B entre otras). Considerando que comparten el mismo mecanismo de transmisión, pero que a diferencia de gonorrea que presenta un corto período de incubación, las otras ITS se caracterizan por presentar manifestaciones clínicas a largo plazo, no se diagnostican al momento de la infección.

La evidencia científica respalda que el uso sistemático y correcto de los preservativos protege eficazmente contra el VIH y reduce el riesgo de infección por gonorrea tanto en hombres como en mujeres ${ }^{11,12}$, lo que reafirma la importancia de esta medida de prevención de las ITS.

Si bien el uso de condón es una de las principales medidas de prevención de las ITS, en nuestro país la declaración de su uso consistente ("siempre") en los últimos 12 meses a nivel nacional es muy bajo (15,6\%). En el grupo de 15 a 19 años y en el de 20 a 24 años el uso consistente sube a 39,4 y $29,6 \%$ respectivamente; no obstante, en estas edades se presentan los mayores riesgos de infección ${ }^{13}$.

En este contexto, y considerando que las co-infecciones de transmisión sexual aumentan la capacidad de infección del VIH, que la menor edad está fuertemente asociada a tener múltiples ITS, que existe un bajo uso de condón en nuestro país, y que al aumentar la gonorrea existe el potencial incremento de otras ITS, el Ministerio de Salud se encuentra estableciendo y ejecutando un plan de medidas específicas de control, prevención y vigilancia dirigido a la red pública y privada de salud.

Además, se debe considerar como refiere el Centers for Disease Control and Prevention (CDC) de Atlanta, Georgia, E.U.A., la infección por $N$. gonorrhoeae se concentra en comunidades específicas, siendo de mayor riesgo el grupo de hombres que tienen sexo con hombres (HSH). En el mundo, la infección por Chlamydia trachomatis y la gonorrea son las dos ITS más comunes entre $\mathrm{HSH}^{8}$.

Según muestran los resultados de vigilancia, $87 \%$ de la infección por $N$. gonorrhoeae se notifica en hombres, que, si bien como se ha revisado, presentan mayor 
sintomatología que las mujeres, este porcentaje podría estar develando una mayor vulnerabilidad en el grupo de HSH. Por tanto, es necesario abordar las estrategias de prevención y control en este grupo poblacional.

Según estudios de comportamiento en HSH en el Reino Unido, se evidenció que a pesar que los participantes sabían sobre la alta prevalencia de gonorrea, declaraban que era poco probable que su comportamiento sexual cambiara y con frecuencia se sentían resignados a repetir las infecciones. El uso de aplicaciones de redes geosociales para organizar encuentros sexuales puede estar contribuyendo a un aumento en las ITS, así como el uso de drogas recreativas, el alcohol y las fiestas sexuales ${ }^{14}$.

En relación al tipo de gonorrea, las infecciones en la faringe y el recto son generalmente asintomáticas ${ }^{15}$, lo que podría explicar el bajo número de casos notificados para esta localización según CIE-10.

Según explica el estudio de un conjunto de brotes de ITS en el Reino Unido, las mejores estrategias de enfrentamiento fueron la combinación del conocimiento sobre la situación de brote y el refuerzo del manejo clínico. Además, el estudio y manejo de los contactos resultó ser eficiente en brotes heterosexuales pequeños, pero resultó ser un desafío en los brotes focalizados en HSH en los que el uso de aplicaciones móviles o el sexo anónimo era común. Se emplearon campañas publicitarias a través de plataformas sociales y organizaciones de la sociedad civil, aunque fue difícil cuantificar su impacto ${ }^{16}$.

Las manifestaciones más graves de la infección por $N$. gonorrhoeae en los RN son la oftalmia y la sepsis, que puede incluir la artritis y meningitis. Otras manifestaciones menos graves incluyen rinitis, vaginitis, uretritis. Si bien hay pocos casos notificados en RN, en el año 2016 se observó un aumento de estos casos, por lo que se debe tener presente que, para evitar la oftalmia neonatal gonocóccica, existe un protocolo de profilaxis.

La profilaxis ocular se justifica porque puede prevenir la oftalmia gonocóccica, tiene un excelente historial de seguridad, es fácil de administrar y es de bajo costo ${ }^{8}$.

Se debe tener presente que la infección gonocóccica en menores de edad debe alertar al equipo de salud, ya que puede ser producto de abuso o violación sexual, el que debe ser denunciado según se establece en Ley $\mathrm{N}^{\circ} 19.696$ Código procesal penal, Ley No 20.207 delitos sexuales contra menores, Ley $N^{o} 19.617$ delito de violación y la Guía Clínica de Abuso Sexual (MINSAL).

La vulnerabilidad en salud se refiere a la falta de protección de grupos poblacionales específicos que presentan problemas de salud particulares, así como a las desventajas que enfrentan para resolverlos, en comparación con otros grupos de población, de manera que la vulnerabilidad en salud es una condición dinámica que resulta de la conjunción de varios determinantes sociales ${ }^{17}$. Como poblaciones más vulnerables se incluye a las personas pertenecientes a pueblos originarios y a los migrantes. $\mathrm{Si}$ bien, según este análisis, estas poblaciones no presentan gran cantidad de infecciones, existe un aumento en la población migrante en el año 2016, lo que no implica que el aumento global de casos de gonorrea esté dado por el incremento en la población extranjera. Además, cabe considerar que esta población presenta barreras de acceso a la atención de salud, particularmente en derecho de salud sexual y reproductiva ${ }^{18}$ y una menor adscripción al sistema de salud. Un $15,7 \%$ no está adscrito a ningún sistema previsional de salud, comparado con $2,7 \%$ de la población nacional ${ }^{19}$.

El 3,6\% de los casos de gonorrea notificados en migrantes es coherente, considerando que los flujos migratorios en Chile han aumentado durante los últimos años, duplicándose el porcentaje desde el CENSO del 2002 (1,2\%), hasta representar en el año 2016: 4\% $(\mathrm{n}=737.046)$ de la población total, según las estimaciones del Departamento de Extranjería y Migración del Ministerio del Interior (DEM). Según distribución de residencia de la población migrante, las regiones de Tarapacá y Antofagasta presentan el mayor porcentaje de extranjeros respecto de su población y a su vez, la R. Metropolitana concentra cerca de $70 \%$ del total de migrantes en Chile según CASEN 2015. Estas son las regiones que concentran la mayor cantidad de casos de gonorrea.

En relación a la declaración de pertenencia a pueblos originarios, se puede advertir que existe escaso registro de esta variable, teniendo en cuenta que la población de pueblos indígenas es de 1.585.680 de acuerdo CASEN 2015. Este notorio sub-registro es coincidente con el alto número de registros nulos en los años 2008 y 2009 (DEIS, 2016) y situación que se advierte en otros registros sanitarios que poseen la variable de pertenencia a pueblos indígenas.

En este contexto, según lo propuesto por la OMS, se debe busca eliminar las inequidades en salud a través de acciones multisectoriales ${ }^{20}$. Es necesario considerar que las personas pertenecientes, tanto a pueblos originarios como migrantes representan un desafío para el sector salud en el abordaje con enfoque de determinantes sociales (DSS) de derechos e interculturalidad. Es así que, en respuesta a esta necesidad, el Ministerio de Salud implementó el Programa Especial de Salud y Pueblos Indígenas desde el año 2000 y en el año 2006 publica la Política de Salud y Pueblos Indígenas ${ }^{21}$. Además, ha desarrollado una estrategia nacional de mejoramiento del registro de la variable de pertenencia a pueblos indígenas. En relación a los migrantes, a partir de 2014 el Ministerio de Salud ha desarrollado una serie de acciones tendientes a disminuir las barreras de acceso. En el año 2016, destaca el Decreto $\mathrm{N}^{\circ} 67$ que permite incorporar al sistema de salud a la población de mayor vulnerabilidad, personas migrantes indocumentadas o sin visa de residencia y 
carentes de recursos ${ }^{22}$. Actualmente, el MINSAL se encuentra desarrollando la "Política de Salud de Migrantes Internacionales".

Dado el actual aumento de la infección por gonorrea, en el concierto internacional existe mayor conciencia del peligro que significa que ésta se vuelva de difícil tratamiento dada la aparición de resistencia antimicrobiana ${ }^{23}$. Es así que $N$. gonorrehoeae, es un microorganismo considerado en la vigilancia de resistencia antimicrobiana. Según lo informado por el laboratorio de referencia nacional (Instituto de Salud Pública), en el período 2010 - junio 2016, de los aislamientos analizados por año, ninguno presentó resistencia a ceftriaxona. Sí se observó una disminución en la sensibilidad a ciprofloxacina, de $67 \%$ en el año 2010 a $37 \%$ en 2015 . Para azitromicina se observó un fenómeno similar, pero de manera más discreta disminuyendo la sensibilidad de $97 \%$ en el año 2010 a $77 \%$ en el 2016. Por otro lado, la mayor sensibilidad a penicilina se observó en el 2010 (15\%), mientras que entre el 2011 y el 2015 este porcentaje fluctuó entre 4 y 8\%. El estudio de cefixime se realiza a partir del año 2016 , observándose que $100 \%$ de las cepas analizadas fueron sensibles. Dados los recientes reportes internacionales de cepas resistentes a cefalosporinas de tercera generación, lo que genera un grave problema terapéutico, es de vital importancia realizar el cultivo del agente, no limitándose a un diagnóstico clínico, y enviarlo al ISP para el estudio de resistencia ${ }^{24}$.

Como conclusión, en Chile desde el año 2000 la gonorrea muestra una tendencia progresiva a la disminución de las tasas; sin embargo, a partir del año 2015 se produjo un aumento de la tasa con relación a los años previos. En el año 2016 se presentó un aumento de $38 \%$ con respecto a la mediana del quinquenio anterior, sobrepasando el número de casos esperados. La infección se concentró principalmente en hombres jóvenes, debido posiblemente a la mayor presentación sintomática respecto a las mujeres. Con relación a la edad, el mayor riesgo se encuentra en el grupo de 15 a 24 años, destacándose que a partir del 2013 la tasa del grupo de 15 a 19 superó al grupo de 25 a 29 años. Según distribución geográfica, las mayores tasas de notificación se presentaron en las regiones del extremo norte y sur del país, siendo las de mayor riesgo las regiones de Tarapacá, Antofagasta, Los Lagos y Aysén.

En este contexto, la situación epidemiológica de gonorrea evidencia una alerta sobre el aumento de casos. A su vez, da una visión sobre el impacto potencial de las estrategias de prevención y manejo de las ITS, además de permitir diseñar, implementar y abogar por el financiamiento de programas e investigaciones ${ }^{6}$. Es fundamental mantener activa y reforzar periódicamente la importancia de la vigilancia epidemiológica de forma oportuna y con calidad en todos los niveles involucrados.

\section{Limitaciones}

Dentro de las limitaciones para la elaboración de este informe, se debe considerar que se trabaja a partir de las notificaciones obligatorias-universales a nivel nacional; no obstante, podría existir sub-notificación de casos, lo que limita conocer la realidad de la infección, además, existen deficiencias en la calidad de los datos ingresados en el ENO, especialmente en la codificación CIE-10 de la gonorrea, en el registro de nacionalidad y declaración de pertenencia a pueblos originarios. Para disminuir la sub-notificación y mejorar la calidad de la información, las Secretarias Regionales Ministeriales (SEREMI) de Salud trabajan en el refuerzo constante de la vigilancia en la red de epidemiología, además de corroborar que se encuentren notificados todos aquellos casos en que se hayan enviado al ISP muestras donde se aisló una $N$. gonorrhoeae.

Por otra parte, dado que el Boletín de Notificación Obligatoria es estándar para todas las enfermedades incluidas en el Decreto $\mathrm{N}^{\circ} 158$, no se cuenta con mayores antecedentes que permitan análisis profundos de la situación epidemiológica como son las conductas, comportamientos de riesgo y otros determinantes sociales.

\section{Recomendaciones}

Se encuentran disponibles en el sitio web del Ministerio de Salud, la siguiente normativa, para vigilancia, manejo y control:

- Circular B51/24 del 23/05/2013. "Vigilancia Epidemiológica de Sífilis y Gonorrea”. Disponible en: http://epi.minsal.cl/wp-content/uploads/2016/05/ CIRCULAR_24_SIFILIS_GONORREA_2013.pdf

- "Norma de manejo y tratamiento de las Infecciones de Transmisión Sexual. Disponible en: http://epi.minsal. cl/wp-content/uploads/2016/07/Norma-103-Infecciones-de-Transmisi\%C3\%B3n-Sexual-1.pdf

- Decreto 206 del 2005. Reglamento sobre Infecciones de Transmisión Sexual. Disponible en: http://epi.minsal.cl/wp-content/uploads/2016/07/REGLAMENTO206-Infecciones-de-Transmision-Sexual-1.pdf

- Otros documentos disponibles en: http://diprece.minsal.cl/programas-de-salud/programa-vih-sida-e-its/ informacion-al-profesional-vih-sida-e-its/

\section{Prevención}

A nivel poblacional e individual la medida más eficiente que permite prevenir la adquisición de las ITS es el uso consistente del condón en todas las relaciones sexuales penetrativas (orales, vaginales y anales). Las prácticas sexuales no penetrativas orales (boca-vulva) y anales (boca-ano), aunque son de menor riesgo para 
gonorrea, si son de riesgo para otras ITS, especialmente virales.

Con el objetivo de cortar la cadena de transmisión, el sistema de salud realiza acciones dirigidas a otorgar diagnóstico precoz y tratamiento oportuno para el paciente (caso índice) y sus contactos sexuales. La norma $\mathrm{N}^{\circ} 187$ de profilaxis, diagnóstico y tratamiento de las ITS indica que, al diagnosticar un caso de gonorrea, el profesional de salud debe entregar información y educación al usuario/a que permita la declaración voluntaria de sus contactos sexuales, adherencia a tratamientos y la adopción de medidas para el autocuidado, evitando así las re-infecciones. Señala, además, la importancia de la obtención de cultivo, como parte del control de la resistencia antimicrobiana.

La concurrencia simultánea de estrategias de prevención primaria (promoción del uso del condón) y prevención secundaria (corte de la cadena de transmisión), en un marco de uso eficiente de los recursos disponibles, permitirían a Chile reducir las nuevas infecciones por gonorrea y por otras ITS, incluido el VIH.

Agradecimientos. Agradecemos a todas las personas que son parte de la red epidemiológica de vigilancia de gonorrea, del Instituto de Salud Pública y de los Programas de ITS en las SEREMIS, Servicios de salud, establecimientos de salud y laboratorios quienes construyen y fortalecen esta vigilancia.

\section{Resumen}

La gonorrea producida por Neisseria gonorrhoeae, es capaz de infectar diferentes tipos de mucosas dependiendo de las prácticas sexuales. Se ubica de preferencia en la uretra del hombre y en el cuello uterino de la mujer, también en el recto y la faringe. Su principal forma de transmisión es por relaciones sexuales no protegidas $\mathrm{y}$, ocasionalmente, a través del conducto del parto en los recién nacidos. Este informe presenta la situación epidemiológica de la gonorrea hasta el año 2016 en Chile. Se realizó un análisis descriptivo de los casos que ingresaron a la vigilancia. Desde el año 2000, se observa una tendencia progresiva a la disminución de las tasas de gonorrea; sin embargo, en el año 2015 y 2016 se produjo un aumento de la tasa con relación al año 2014. En el año 2016 se presentaron 2.039 casos, lo que representa un incremento de $38 \%$ con respecto a la mediana del quinquenio anterior (1.473 casos). En relación con la edad de los casos, el mayor riesgo se encontró en el grupo de 15 a 24 años, destacándose que, a partir del año 2013, la tasa del grupo de 15 a 19 años superó al grupo de 25 a 29 años. Según distribución geográfica, las mayores tasas de notificación se presentaron en las regiones del extremo norte y sur del país, siendo las de mayor riesgo las regiones de Tarapacá, Antofagasta, Los Lagos y Aysén.

\section{Referencias bibliográficas}

1.- Organización Panamericana de la Salud (OPS); Organización Mundial de la Salud (OMS); Programa Conjunto de las Naciones Unidas sobre el VIH/Sida (ONUSIDA). Pautas Vigilancia de las infecciones de transmisión sexual.1999 Disponible en: http://www.who.int/ hiv/pub/sti/en/cds_csr_edc_99_3sp.pdf.

2.- Conde-González $\bar{C}$, Uribe Salas F. Gonorrea: la perspectiva clásica y la actual. Salud pública Méx 199739 (6): 543-79.

3.- Centers for Disease Control and Prevention. 2011 Sexually Transmitted Diseases Surveillance. USA. 2012. Disponible en: https://www.cdc.gov/std/stats12/surv2012. pdf.

4.- Cano S, Fuentes M, Ballesteros J, Clavo P, Menéndez B, Del Romero J. Diagnósticos de gonorrea en un centro de infecciones de transmisión sexual y su relación con el virus de la inmunodeficiencia humana y otras infecciones de transmisión sexual, Madrid 2005. Enferm Infecc Microbiol Clin 2009; 27 (6): 338-41.

5.- Ministerio de Salud de Chile. Circular B51/24 Vigilancia epidemiológica de sífilis y gonorrea.
Santiago, 2013. Disponible en: http://epi. minsal.cl/wp-content/uploads/2016/05/ CIRCULAR_24_SIFILIS_GONORREA_2013. pdf.

6.- Newman L, Rowley J, Vander Hoorn S, Wijesooriya NS, Unemo M, Low N, et al. Global estimates of the prevalence and incidence of four curable sexually transmitted infections in 2012 based on systematic review and global reporting. PLoS ONE 2015; 10 (12): e0143304. Disponible en: https://doi. org/10.1371/journal.pone.0143304.

7.- $\quad$ Da Ros, Da Silva. Global epidemiology of sexually transmitted diseases. Brazil. Asian J Androl 2008; 10 (1) 110-4. Disponible en: 10.1111/j.1745-7262.2008.00367.x.

8.- Centers for Disease Control and Prevention. Sexually Transmitted Diseases Treatment Guidelines, 2015. MMWR Morb Mortal Wkly Rep. Recommendations and Reports. 2015; 65 (3). Disponible en: http://www.cdc.gov/std/ $\operatorname{tg} 2015 /$ tg-2015-print.pdf .

9.- Kalichman SC, Pellowski J, Turner C. Prevalence of sexually transmitted coinfections in people living with HIV/AIDS: systematic review with implications for using HIV treatments for prevention. Sex Transm
Infect 2011; 87 (3): 183-90. doi: 10.1136/ sti.2010.047514.

10.- Lie P, Weaver Ch, Van Damme M, Baseline L. Factors associated with incident HIV and STI in four microbicide trials. Feldblum. Sex Transm Dis 2010; 37 (10): 594-601 doi: 10.1097/ OLQ.0b013e3181e15f0b.

11.- Warner L, Stone K, Macaluso M, Buehler J, Austin H. Condom use and risk of gonorrhea and chlamydia: A systematic review of design and measurement factors assessed in epidemiologic Studies. Sex Transm Dis 2006; 33 (1): 36-51.

12.- Holmes K, Levine R, Weaver M. Effectiveness of condoms in preventing sexually transmitted infections. Bull World Health Organ. 2004; 82 (6): 454-61. Disponible en: http://www.ncbi. nlm.nih.gov/pmc/articles/PMC2622864/

13.- Dpto. de Epidemiología, DIPLAS_MINSAL Encuesta Calidad de vida y salud 2015 2016. Disponible en: http://epi.minsal.cl/ wp-content/uploads/2017/06/Resultados Abril2017_ENCAVI_2015-16_Depto_ Epidemiolog\%C3\%ADa_MINSAL.pdf.

14.- Payne, D Lawrence, S Soni, Llewellyn C, Dean $\mathrm{G}$. Investigating factors for increased gonorrhoea re-infection in men who have 
sex with men attending a genitourinary clinic: a qualitative study. Intern J STD and AIDS; 2016. doi: 10.1177/095646241 6677916.

15.- Chow E, Camilleri S, Ward C H, Huffam S, Chen M, Bradshaw C, et al. Duration of gonorrhoea and chlamydia infection at the pharynx and rectum among men who have sex with men: a systematic review. Sexual Health 2016; 13: 199-204. doi: 10.1071/ SH15175.

16.- Loftus H, Mackenzie K, Simms I, Cassell K. Management of STI outbreaks. What can we learn from each other? A qualitative study in the UK. Sex Transm Infect 2016; 92 (Suppl 1): A1-A106. Disponible en: 10.1136/ sextrans-2016-052718.253.

17.- Juárez-Ramírez C, Márquez-Serrano M, Salgado de Snyder N, Pelcastre-Villafuerte B E, Ruelas-González M G, Reyes-Morales H.
La desigualdad en salud de grupos vulnerables de México: adultos mayores, indígenas y migrantes. Rev Panam Salud Publica 2014; 35 (4): 284-90.

18.- Chile. Ministerio de Desarrollo Social. Encuesta de caracterización Socio económica Nacional 2015. http://observatorio. ministeriodesarrollosocial.gob.cl/casenmultidimensional/casen/casen_2015.php.

19.- Liberona N. La frontera cedazo y el desierto como aliado. Prácticas institucionales racistas en el ingreso a Chile. Polis Revista Latinoamericana 2015; 14 (42): 143-65. http://dx.doi.org/10.4067/S071865682015000300008.

20.- Organización Mundial de la Salud (OMS), Comisión sobre Determinantes Sociales de la Salud. Subsanar las desigualdades en una generación: alcanzar la equidad sanitaria actuando sobre los determinantes sociales de la salud. Informe final. Ginebra: OMS; 2008. Disponible en: whqlibdoc.who. int/hq/2008/ WHO IER CSDH 08.1 spa.pdf.

21.- Chile. Ministerio de Salud. Política de Salud de pueblos indígenas. Resolución exenta $\mathrm{N}^{\mathrm{o}} 91 \mathrm{del}$ 10/02/2006.

22.- Chile. Ley Modifica decreto No 110 de 2004, del Ministerio de Salud, que fija circunstancias y mecanismos para acreditar a las personas como carentes de recursos o indigentes. Disponible en: https://www.leychile.cl/Navegar ?idNorma=1088253\&idVersion=2016-03-10.

23.- Creighton S. Gonorrhoea. BMJ Clinical Evidence. 2014; 02:1604. Disponible en: https://www.ncbi.nlm.nih.gov/pmc/articles/ PMC3931440/pdf/2014-1604.pdf.

24.- Instituto de Salud Pública. Vigilancia de Neisseria gonorrhoae Chile 2010-Junio 2016. Disponible en: http:/www.ispch.cl/sites/ default/files/Boletin\%20Gono-Final.pdf. 